\title{
Enfoque para la integración de sistemas de gestión en empresas de servicios del sector hidrocarburos*
}

\section{Approach to integrating management systems in the hydrocarbon sector service companies}

Recibido: 02 de diciembre de 2014

Revisado: 22 de mayo de 2015

Aceptado: 27 de junio de 2015

Félix Antonio Rivera Bolivar**

Universidad Santo Tomás - Icontec

\section{RESUMEN}

La integración de los sistemas de gestión en las organizaciones constituye una ventaja competitiva en entornos cambiantes y cada vez más exigentes. Este estudio se dirige a generar un enfoque para la integración de los sistemas de gestión (calidad, seguridad, salud y ambiente) que pueda ser aplicado en organizaciones de servicios del sector de hidrocarburos. Se obtiene información de este sector para entender el entorno de las empresas que prestan servicios en él, de la cadena de valor en la producción de hidrocarburos y de las principales actividades del sector servicio petrolero en Colombia; se revisan los requisitos en cuanto a calidad, seguridad, salud y ambiente de las dos principales empresas contratantes del sector hidrocarburos y se revisan modelos de integración de sistemas de gestión. Además, se entrevista a los funcionarios responsables de seis empresas que prestan servicios al sector y, finalmente, con los resultados obtenidos se plantea el modelo GOLD (gestión organizacional con liderazgo directivo) como mecanismo para facilitar la integración de los sistemas de gestión en las organizaciones de servicios del sector de hidrocarburos.

* Artículo de investigación.

* Ingeniero Químico. Especialista en Seguridad y Prevención de Riesgos Laborales. Magíster en Calidad y Gestión Integral. Correo electrónico: fariverab@yahoo.com 
El modelo GOLD se fundamenta en el liderazgo y apoyo de la alta dirección y en la gestión organizacional que busca comprender el entorno y los factores políticos, económicos, sociales, tecnológicos y ambientales que presentan oportunidades, amenazas y riesgos para la organización. Se analiza la evolución que ha tenido el liderazgo en el sector de los servicios y se indican lineamientos para el desarrollo del sistema integrado de gestión (SIG).

Palabras clave: calidad, partes interesadas, sistema integrado de gestión, sistema de gestión. management that seeks to understand the environment and the political, economic, social, technological, and environmental factors that present opportunities, threats and risks to the organization. The leadership evolution that has taken in the sector of services and guidelines for the development of integrated management system (IMS) are indicated.

Keywords: Quality, stakeholders, integrated management system, management system.

\section{INTRODUCCIÓN}

Las organizaciones prestadoras de servicios en nuestro país están mejorando su gestión para responder a las exigencias de los mercados, con procesos de implementación y certificación de normas técnicas sobre sistemas de gestión. Las normas técnicas de gestión más implementadas son la NTC-ISO 9001 (2008) para el sistema de gestión de la calidad, la NTC-ISO 14001 (2004) para el sistema de gestión ambiental y la NTCOHSAS 18001 (2007) para el sistema de gestión en seguridad y salud ocupacional. A pesar de las múltiples posibilidades que ofrece la integración de sistemas de gestión para el mundo empresarial, las organizaciones prestadoras de servicios en Colombia no se han podido beneficiar, en general, de estas ventajas, dado el fuerte paradigma cultural por el cual se piensa que la integración de sistemas de gestión es un proceso costoso y dispendioso, indicado solamente para las empresas más grandes del sector y para multinacionales con grandes capacidades operativas.

En los últimos años, la actividad petrolera en el país ha crecido paulatinamente y con ello la preocupación acerca del desempeño del sector. Si bien todavía no se puede hablar de Colombia como una economía petrolera, lo cierto es que se trata de una economía con una producción importante de crudo, en la cual dicha 
actividad resulta estratégica por su participación en el producto interno bruto (PIB). En el año 2014, la producción petrolera represento entre el 5 y $6 \%$ del PIB nacional (Fedesarrollo, 2015).

Teniendo en cuenta la importancia que ha tomado el sector de hidrocarburos para la economía nacional, las grandes contratantes y operadoras del sector realizan mayores exigencias en los temas de calidad, medio ambiente, seguridad y salud ocupacional a las empresas que les prestan servicios, por esto las empresas contratistas han venido implementando y certificando los sistemas de gestión, con el propósito de obtener mayor participación en contratos que les ofrecen las operadoras; además, para mejorar su gestión han emprendido estrategias de integración de una forma empírica.

Para analizar cómo las empresas de servicios han venido implementando y manteniendo los sistemas de gestión, en el desarrollo del proyecto se contempló visitar seis empresas y realizar el análisis en ellas con el fin de establecer un enfoque para la integración de los sistemas de gestión de calidad, medio ambiente y seguridad y salud ocupacional, a la luz de sus respectivas normas técnicas (NTC-ISO 9001, NTC-ISO 14001 y la NTC-OHSAS 18001), con el propósito de que las empresas de servicios del sector de hidrocarburos puedan cumplir con las exigencias de sus clientes nacionales 0 internacionales en esta materia y puedan maximizar el logro de los beneficios potenciales de la integración.

Las empresas contratistas del sector hidrocarburos han venido exigiendo sistemas de gestión de calidad, medio ambiente y seguridad y salud ocupacional con el fin de garantizar un buen servicio y prevenir accidentes laborales e impactos ambientales.

Se aborda el tema de la integración de los sistemas de gestión partiendo de los lineamientos de las normas en los temas de calidad, seguridad, salud ocupacional y gestión ambiental, con un enfoque de gestión integral.

Las empresas de servicios pertenecientes al sector de hidrocarburos deben atender los requerimientos de sus clientes y la mayor competitividad de las empresas del sector. El aumento de los requerimientos legales y de las exigencias han llevado a que muchas empresas de servicios se orienten hacia una nueva perspectiva organizacional que involucra generar confianza a sus clientes y partes interesadas con respecto a la calidad de sus productos y en temas de salud, seguridad y medio ambiente.

Friedman (2009) menciona que el paradigma de desarrollo sostenible se hace cada vez más un tema de negocio, esto implica una transición en las empresas en la que los procesos se enfoquen a la creación de valor económico, valor para el ambiente natural y el ambiente social. "El foco está orientado a un cambio en las empresas hacia la dirección integrada, y la organización tiene que sostener el negocio en mercado competitivo" (Friedman (2009), aspecto que genera nuevos retos en un entorno cambiante.

Haciendo referencia a la dirección integrada y su aporte para generar organizaciones más competitivas, los docentes investigadores de la Universidad Santo Tomás - Icontec, Guillermo Peña y Fabio Tejada, en su artículo "Reflexiones sobre las características constitutivas de la gestión integral" (Peña y Tejada, 2009, p. 92) plantean que la organización debe gestionarse integralmente de manera coherente, sistémica y sistemáticamente para responder a las exigencias del mercado y de los clientes en cuanto a sus expectativas y requisitos, por esto, una dirección en la que se gestionen integralmente los sistemas de gestión puede responder de manera más efectiva a las necesidades y expectativas de las partes interesadas. 
Durante el proceso de planteamiento del problema, surgieron reflexiones que permitieron generar el siguiente interrogante de investigación:

¿Podría la integración de los sistemas de gestión en empresas de servicios del sector hidrocarburos generar una mayor optimización de los recursos al gestionar los diferentes sistemas implementados y hacer más competitiva la organización?

La importancia de este trabajo de investigación se manifiesta en su utilidad e interés para las empresas de servicios en el sector de hidrocarburos porque les permitirá mantener una imagen competitiva, facilitar la administración de los procesos, reducir los costos por documentación, optimizar las actividades de planeación, auditoría y revisión del sistema de gestión, además de mejorar el desarrollo organizacional con la integración de los sistemas de gestión y la gestión estratégica de las organizaciones.

Como objetivo general, se espera lograr generar un enfoque para la integración de los sistemas de gestión (calidad, seguridad, salud y ambiente) que pueda ser aplicado en empresas de servicios del sector de hidrocarburos de Colombia.

Los objetivos específicos del proyecto se orientan a realizar el análisis del entorno por medio del análisis PESTA a una empresa que presta servicios en el sector de hidrocarburos; a revisar los requerimientos en los temas de calidad, seguridad, salud y ambiente exigidos en los procesos de licitación a las empresas que prestan sus servicios; a elaborar un diagnóstico de la situación actual de los sistemas de gestión frente a la integración, tomando como muestra seis empresas que prestan servicios al sector hidrocarburos y que están certificadas en las normas NTC-ISO 9001, NTC-ISO 14001 y NTC-OHSAS 18001; y a establecer un modelo que permita dar lineamientos para facilitar la integración de un sistema de gestión (calidad, seguridad, salud y ambiente) el cual pueda ser aplicado en empresas de servicios del sector de hidrocarburos.

\section{METODOLOGÍA}

Teniendo en cuenta los objetivos de la investigación, el presente trabajo se enmarcó dentro de la metodología descriptiva, siguiendo los lineamientos expuestos por César Augusto Mario Bernal en su libro Metodología de la investigación (Bernal, 2006, pp. 112-113), ya que se buscó realizar una reseña de las características o rasgos de un fenómeno de estudio para establecer un instrumento para la integración de los sistemas de gestión en empresas del sector hidrocarburos.

La presente investigación contempla varias fases:

- FASE 1, orientada a buscar información para caracterizar y entender el entorno de una de las empresas que prestan servicios del sector hidrocarburos, actividad que, mediante el análisis PESTA, busca realizar el análisis de entorno. En el marco teórico se explica la cadena de valor en la producción de hidrocarburos, se explican las principales actividades del sector servicios petrolero en Colombia y su evolución.

- FASE 2, exploratoria, para revisar, por medio de los procesos licitatorios, los requisitos en cuanto a calidad, seguridad, salud y ambiente de empresas contratantes del sector hidrocarburos y revisar modelos de integración de sistemas de gestión.

- FASE 3, descriptiva, con el interés de elaborar un diagnóstico de la situación actual de los sistemas de gestión frente a la integración para las empresas de servicios del sector de hidrocarburos certificadas. En esta fase se plantea realizar la visita a seis 
empresas y la entrevista a las personas responsables de los sistemas de gestión.

- FASE 4, en la cual se aborda el análisis de los resultados obtenidos. Se plantea como resultado un modelo conceptual que permita dar los lineamientos para facilitar la integración de los sistemas de gestión.

La población objeto de este estudio fueron seis empresas del sector de servicios para el sector de hidrocarburos, cinco en la ciudad de Bogotá y una en Chía, certificadas en las normas NTC-ISO 9001:2008, NTCISO 14001:2004 y NTC-OHSAS 18001:2007.

La selección de la muestra se sustentó teniendo en cuenta el muestreo no probabilístico de tipo intencional o de criterio. Este tipo de muestreo se caracteriza por un esfuerzo deliberado de obtener muestras mediante la inclusión, en la muestra, de grupos típicos; por lo tanto, se tomaron empresas del sector servicios que estaban certificadas y en las cuales se pudo entrevistar al responsable de la implementación del sistema de gestión (Hulley y Cummings, 1993).

\section{RESULTADOS Y ANÁLISIS}

De acuerdo con los objetivos planteados para este proyecto, y como resultado de la primera fase, la cual estuvo orientada en buscar información para caracterizar y entender el entorno de una de las empresas que prestan servicios del sector hidrocarburos, se pudo ver que este sector es pionero en la introducción de métodos de control para garantizar que las empresas que les presten servicios cumplan sus requisitos y expectativas. El sector de los hidrocarburos se ha caracterizado por requerir una fuerza de trabajo especializada, que cuente con equipos de avanzada tecnología (Fedesarrollo, 2015) y por la incorporación, de parte de las empresas, de valor agrado a sus servicios, lo que le ha hecho posible responder a las necesidades cambiantes de la industria del petróleo, razón por la cual ha permitido el ingreso de empresas de servicios de otros países. Por esto, y ante la necesidad de aumentar la producción de hidrocarburos en el país, las empresas interesadas en ingresar al mercado de los servicios petroleros deben conocer los diferentes requisitos exigidos por los clientes potenciales que van a utilizar sus servicios y conocer el entorno del sector, el cual se presenta de manera resumida a continuación; luego se dará un ejemplo con base en una empresa que formó parte de la muestra.

\section{Entorno sector hidrocarburos}

En el contexto nacional, dos de los factores que más han incidido en el crecimiento del sector han sido el aumento de la participación privada en las actividades petroleras y el fomento a la inversión extranjera directa (IED). La participación del sector petrolero en la IED total aumentó $14 \%$ de 2003 a 2013, pasando de $16 \%$ a $30 \%$. Como consecuencia de lo anterior, se dio un aumento considerable del número de pozos perforados, el cual pasó de menos de 30 en 2003 y 2004 a más de un centenar por año entre 2010 y 2013 (Unidad de Planeación Minero Energética (2015).

El fuerte incremento de la producción del petróleo en el período 2003-2013, consecuencia, en gran parte, del dinamismo del precio internacional, ha implicado un aumento de su participación dentro del PIB y las exportaciones nacionales. Sin embargo, el aumento de la importancia del sector de hidrocarburos en la economía no se da de forma homogénea. Por una parte, las características de la producción petrolera está concentrada en la mano de obra calificada y se caracteriza por encadenamientos productivos escasos y débiles con otros sectores. Por el otro lado, la gran capacidad de atracción de capitales y vocación exportadora hace 
que la balanza externa del país dependa del comportamiento de esta actividad, al tiempo que la fuerte contribución a las cuentas fiscales, por medio de los impuestos y de la transferencia de los dividendos de Ecopetrol S. A., ha representado un aumento de la dependencia fiscal de la evolución del sector (Unidad de Planeación Minero Energética (2015).

El sector hidrocarburos aporta cerca del $5 \%$ del PIB y algo más del $20 \%$ de los ingresos corrientes del Gobierno nacional. Su importancia para el desarrollo de las regiones es notable, en la medida en que los ingresos por regalías constituyen una fuente de recursos para los gobiernos territoriales (Fedesarrollo, 2015).

Un aspecto que ha marcado el desempeño del sector de hidrocarburos ha sido la evolución del marco institucional con la creación de la ANH y la capitalización de Ecopetrol S. A., que vendió 10,1 \% de sus acciones al público, lo cual le generó ingresos importantes para invertir y expandir sus operaciones.

El sector de hidrocarburos es intensivo en capital, los procesos de perforación, extracción y refinación requieren de maquinaria de gran envergadura y costo, mientras que necesitan una inversión relativamente pequeña en fuerza laboral. Por ende, la actividad petrolera provee principalmente empleo formal y que se caracteriza por empleos de alto riesgo.

\section{Análisis de entorno empresa sector hidrocarburos}

Para recopilar la información que permitiera establecer el análisis de entorno en una empresa de la muestra tomada (MARENFOX S.A.), se realizó la entrevista al gerente general y al responsable del sistema de gestión.
MARENFOX S. A. es una empresa dedicada a la prestación de servicios de workover para el sector de hidrocarburos, la cual se tomó de muestra para realizar el análisis de contexto que se describe a continuación.

\section{Análisis político legal}

Servicio con regulaciones por medio de la legislación y los entes de control, como la Agencia Nacional de Hidrocarburos (ANH).

Para dar cumplimiento a los requisitos legales, MARENFOX S.A. tiene un procedimiento para identificar y tener acceso a los requisitos legales de HSEQ, el cual contiene las leyes y los reglamentos asociados a la actividad económica que ejerce. De la misma manera, tiene como objetivo establecer el mecanismo para identificar, controlar y mantener actualizados los requerimientos legales aplicables a su actividad.

\section{Análisis económico}

Perspectivas de crecimiento dadas por las inversiones en el sector hidrocarburos y favorecidas por la calificación de grado de inversión otorgadas a Colombia por la Moody's Standard \& Poors.

\section{Análisis sociocultural}

Generación de empleo en el desarrollo de cada uno de los proyectos y apoyo a las comunidades cercanas para dar trabajo a mano de obra menos calificada con niveles de competencia media.

\section{Análisis tecnológico}

Equipo de workover montado sobre ruedas y con sistemas hidráulicos para levantamiento y nivelación. Tecnología costosa con altos estándares de seguridad. 


\section{Análisis ambiental}

Legislación reciente en materia ambiental para realizar una disposición adecuada de residuos generados en la actividad económica. Nuevos costos asociados al cumplimiento legal ambiental.

\section{Análisis interno}

\section{Sistemas y modelos adoptados por la organización}

La organización ha realizado la implementación de los siguientes sistemas de gestión: ISO 9001:2008, ISO 14001:2004 y OHSAS 18001:2007.

\section{Capacidades organizacionales}

La organización dispone de dos sedes. El mantenimiento de los sistemas de gestión es liderado por un coordinador HSEQ, y cada actividad de workover cuenta con un supervisor HSEQ de campo.

Para garantizar una adecuada competencia de su personal, la compañía estableció las competencias definidas en términos de educación, experiencia, habilidades y entrenamiento, apropiadas para cada cargo, estableciendo asílas competencias para todo el personal.

\section{Análisis DOFA}

\section{Debilidades}

Débil integración de los sistemas de gestión, ausencia de estandarización de tarifas, falta de apoyo comercial en otras regiones del país (imagen corporativa, medios de comunicación), falta análisis de la competencia, fallas en la comunicación con los clientes y dificultad en el manejo eficiente de la información.

\section{Oportunidades}

Crecimiento continuo del mercado, barreras de entrada para nuevos competidores, reglamentación del sector, desarrollo tecnológico, procesos licitatorios, invitaciones privadas y alianzas con inversionistas extranjeros (joint venture).

\section{Fortalezas}

Certificación en sistemas de gestión, trayectoria y experiencia en el mercado, experiencia en participación en licitaciones, diversidad de equipos para prestar el servicio al cliente.

\section{Amenazas}

Nuevos requisitos de los clientes, alta competencia, seguridad pública en zonas de trabajo, competencia desleal, disminución del precio del petróleo, situación sociopolítica de los países petroleros de Oriente.

\section{Grupos de interés}

Este análisis se enfocó en determinar sus necesidades 0 requerimientos.

\section{Clientes}

Cumplimiento y calidad en la prestación del servicio.

\section{Entidades reguladoras}

Cumplimiento de las normas legales aplicadas al sector (Ministerio de Minas, Ministerio de Trabajo y Ministerio de Ambiente y Desarrollo Sostenible).

\section{Proveedores}

Cumplimiento de pago a proveedores. Desarrollo con la organización.

\section{Socios}

Rentabilidad y utilidad por la los socios. Proyección de cambio generacional. 


\section{Empleados}

Cumplimiento en el pago de salario, prestaciones, crecimiento y desarrollo, calidad de vida laboral y de los estándares de seguridad y salud ocupacional.

\section{Establecimiento de perspectivas}

\section{Perspectiva finaciera}

Lograr un crecimiento sostenido y rentable de la organización.

\section{Perspectiva con el cliente}

Incrementar la satisfacción de los clientes. Aumentar el volumen de servicios por cliente.

\section{Perspectiva del medio ambiente}

Mitigar los impactos ambientales generados por las actividades.

\section{Perspectiva de seguridad y salud}

Lograr un ambiente sano, identificando los peligros y estableciendo estrategias para la minimización de los riesgos.

\section{Innovación y aprendizaje}

Realizar investigación de nuevas alternativas que empleen tecnología mejorada para optimizar la prestación del servicio.

\section{Misión y visión de MARENFOX S.A.}

\section{Misión}

Contribuir al desarrollo de la industria petrolera en la prestación de servicios de workover, buscando potenciar la capacidad de sus actividades con el cumplimiento de los estándares de calidad, seguridad, salud y medio ambiente.

\section{Visión}

Optimizar sus servicios y lograr liderazgo en el sector petrolero. Consolidarse como una empresa reconocida por su competitividad y eficiencia en el suministro de equipos, herramientas y personal calificado, cumpliendo los estándares de calidad, seguridad, salud ocupacional y ambiente.

\section{Requerimientos en los temas de calidad, seguridad, salud y ambiente exigidos a las empresas que prestan servicios en el sector hidrocarburos}

Cuando las empresas de servicios petroleros están interesadas en ingresar al mercado, deben conocer los diferentes requisitos exigidos por los clientes potenciales que van a utilizar sus servicios. En este caso particular, y como objetivo del proyecto de investigación, se analizarán los requisitos de Ecopetrol y Pacific, las dos más grandes del país.

Con respecto a los temas de calidad, seguridad, salud y ambiente solicitados por Ecopetrol en su Manual de contratación, se presentan las normas y los procedimientos bajo los cuales se celebran tanto los procesos de selección de contratistas como los contratos que Ecopetrol requiera para el desarrollo de su objeto social (Ecopetrol, 2012). Además, se establecen los requerimientos HSE en la guía foco que Ecopetrol (2013) exige para poder participar en sus procesos licitatorios, en los que se analizan aspectos como capacidad legal, capacidad técnica, capacidad financiera, financiación, aspectos de HSEQ, manifestación oportuna de intención de participar o escogencia mediante sorteo público o invitación a participar, garantía de seriedad de la propuesta cuando se considere conveniente, visita 
al sitio de ejecución del contrato y presentación de la propuesta, entre otros aspectos que se consideren oportunos para la ejecución del contrato.

La guía establece los requisitos de HSE que se consideran en cada fase del proceso de contratación (planeación, selección de contratista, ejecución, terminación y liquidación del contrato) en función de los riesgos que se encuentren asociados a las actividades de ejecución del objeto y alcance del contrato.

En cuanto a Pacific Rubiales Energy, como segundo mayor productor de gas y petróleo del país, en el Anexo HSEQ Pacific Rubiales Energy se establecen los requisitos mínimos de salud ocupacional, seguridad industrial, medio ambiente, calidad (HSEQ) que deben cumplir los contratistas y sus subcontratistas. Estas especificaciones forman parte integral del contrato $\mathrm{u}$ orden de servicios que debe ejecutar el contratista, pero no reemplazan las obligaciones de ley que sean aplicables.

Pacific Rubiales Energy cuenta con estándares, cuyos requisitos y procedimientos se consideran aplicables a las actividades desarrolladas en el marco de los contratos suscritos con sus contratistas. Los requisitos de salud ocupacional, seguridad industrial, medio ambiente, calidad (HSEQ) forman parte integral de la invitación a proponer, la solicitud de oferta, el contrato y/o la respectiva orden de servicios o de compra. Pacific Rubiales Energy mantiene un sistema de gestión bajo las directrices de las normas técnicas ISO 9001, ISO 14001 y OHSAS 18001, en el que se considera a las actividades de los contratistas como parte de las actividades de la organización.

\section{Diagnóstico de los sistemas de gestión en empresas de servicios}

Para la elaboración del diagnóstico de los sistemas de gestión en empresas que prestan servicios al sector hidrocarburos, se tomaron seis organizaciones que estuviesen certificadas en las normas NTC-ISO 9001, NTC-ISO 14001 y NTC-OHSAS 18001; esta selección fue intencional, ya que en ellas se pudo aplicar la entrevista a la persona responsable del mantenimiento y la implementación del sistema de gestión.

Al aplicar la entrevista se obtuvieron los siguientes resultados:

Fig̉ura 1. Integración sistemas de gestión que tiene implementados

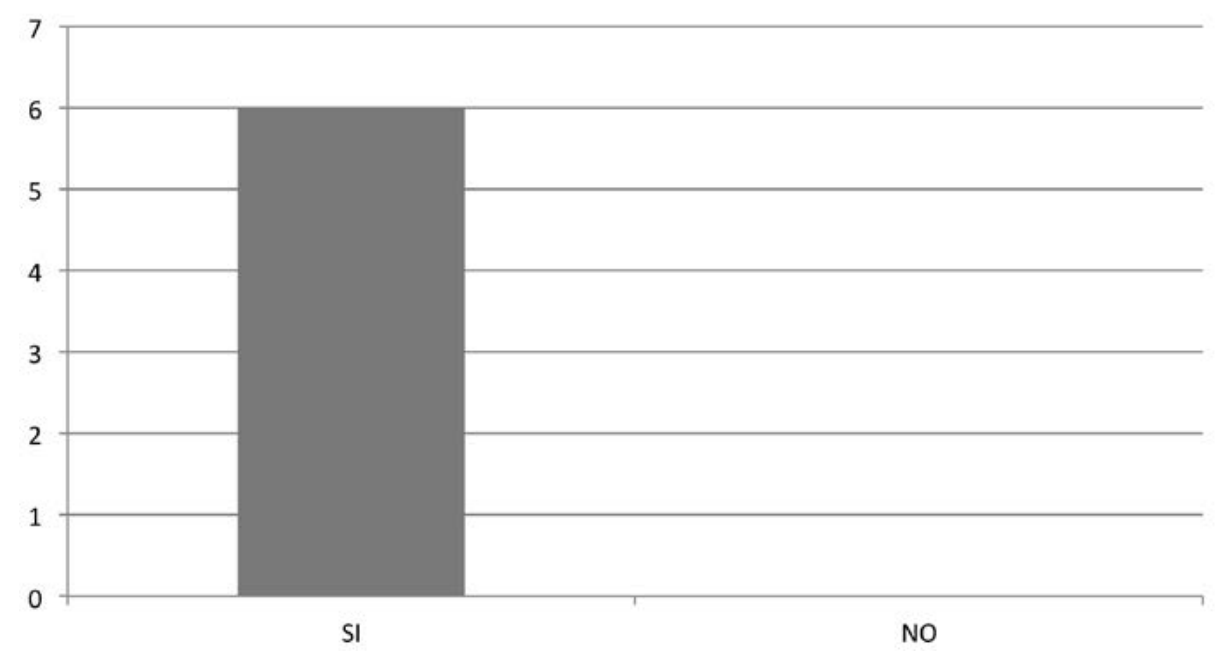

Fuente: autor. 
Según la figura 1, a la pregunta çha integrado la organización los sistemas de gestión que tiene implementados?, las seis empresas a las cuales se les realizó la entrevista manifestaron que sí lo han hecho.

La siguiente consulta planteaba lo siguiente: Si la respuesta a la anterior pregunta es afirmativa, ¿qué motivo llevó a la organización a la integración de los sistemas de gestión? Como se observa en la figura 2, una empresa manifestó que el motivo de la integración fue simplificar procesos, dos se refirieron a la participación en el mercado y tres dijeron hacerlo por ser un requisito del cliente.

Figura 2. Motivo que llevó a la integración de los sistemas de gestión

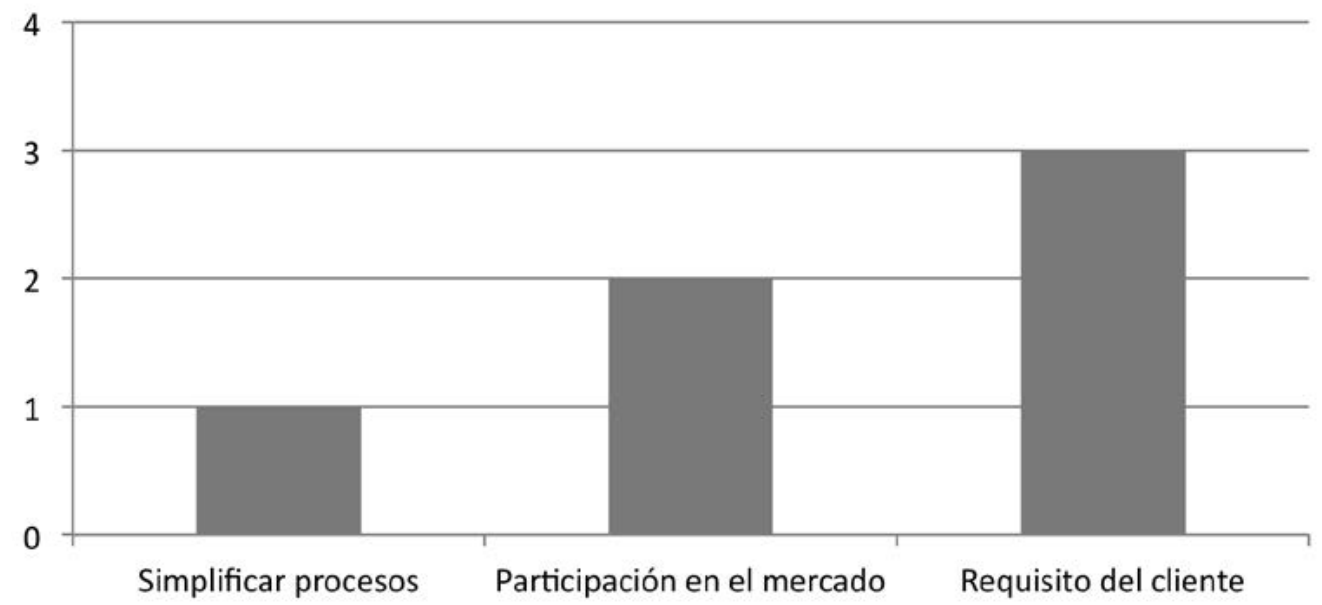

Fuente: autor.

Figura 3. Enfoque que utilizó la organización para la integración de los sistemas de gestión

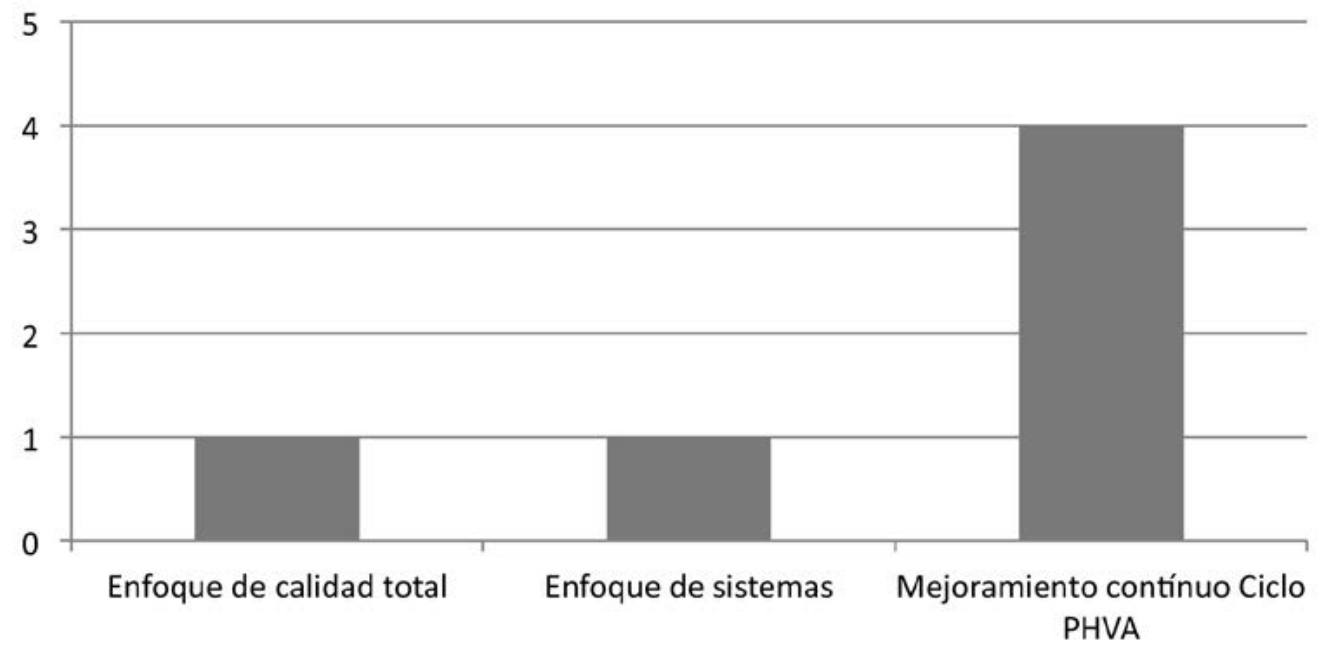

Fuente: autor. 
Con respecto al enfoque o modelo que la organización utilizó para la integración de los sistemas de gestión, de la figura 3 se concluye que cuatro empresas utilizaron el modelo de mejoramiento continuo, una empresa empleó el enfoque de calidad total y la otra hizo uso del enfoque de sistemas.
De acuerdo a la figura 4, cuatro empresas refirieron que su sistema de gestión si está alineado con la planeación estratégica y dos respondieron que no.

Figura 4. Sistema de gestión alineado con la planeación estratégica

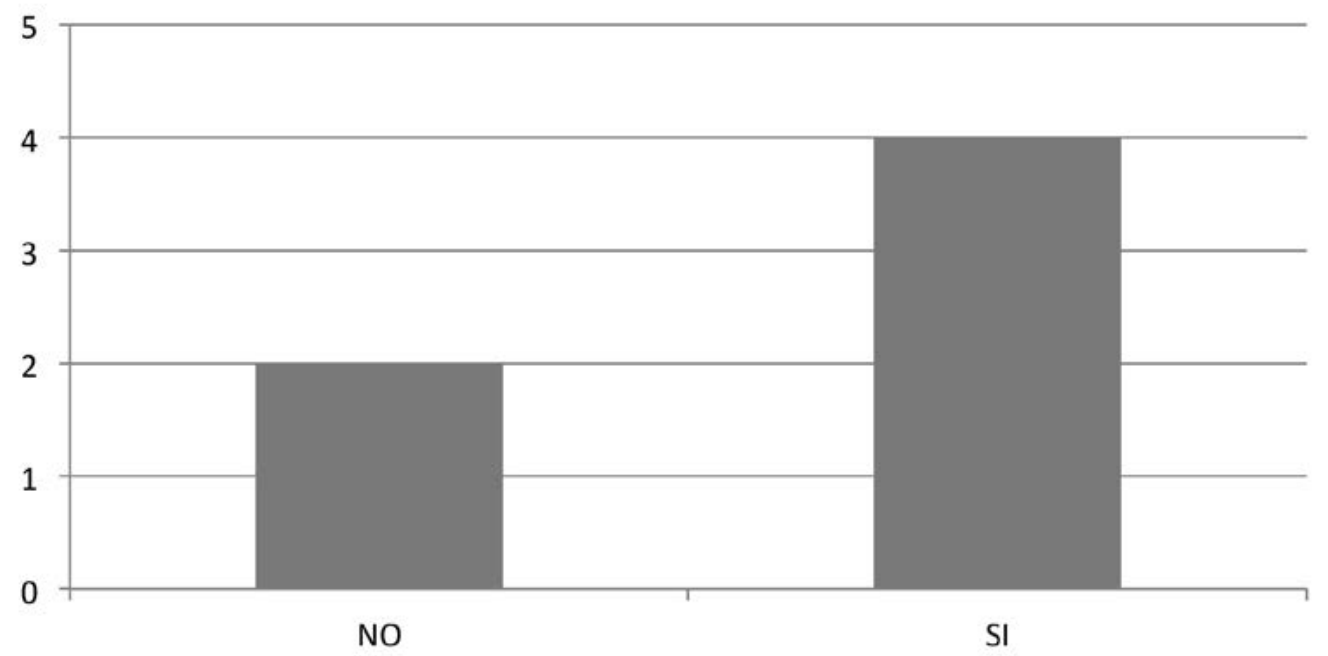

Fuente: autor.

Figura 5. Ventajas de la integración de los sistemas de gestión

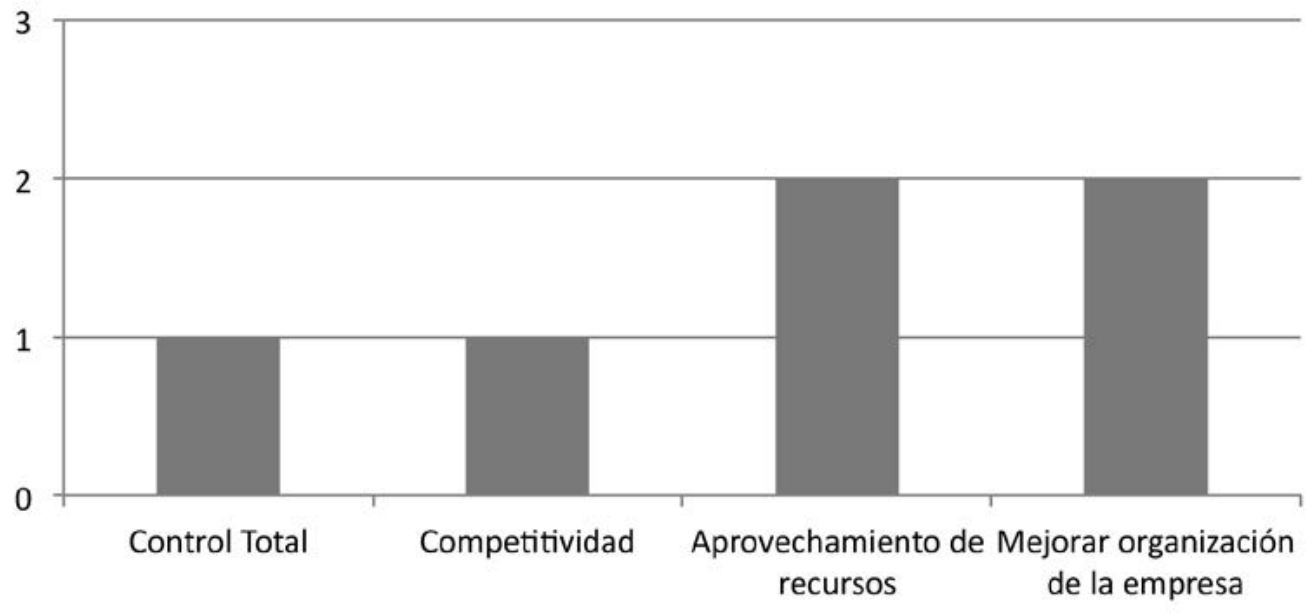

Fuente: autor. 
Con respecto a las ventajas se pueden obtener en la implementación de la integración de los sistemas de gestión, de acuerdo a la figura 5, dos empresas manifestaron que la ventaja de la integración es la mejora en la organización de la empresa, otras dos mencionaron el aprovechamiento de recursos, una refirió el control total y otra dijo que la ventaja es la competitividad.
Con relación a las desventajas que se pueden generar de la implementación de la integración de los sistemas de gestión, según la figura 6, dos empresas indicaron que la desventaja es el aumento de costos, una se refirió a la sobrecarga en la documentación, otra a la falta de cultura y las dos empresas restantes expresaron que no hay ninguna desventaja.

Figura 6. ¿Qué desventajas se pueden generar de la implementación de la integración de los sistemas de gestión?

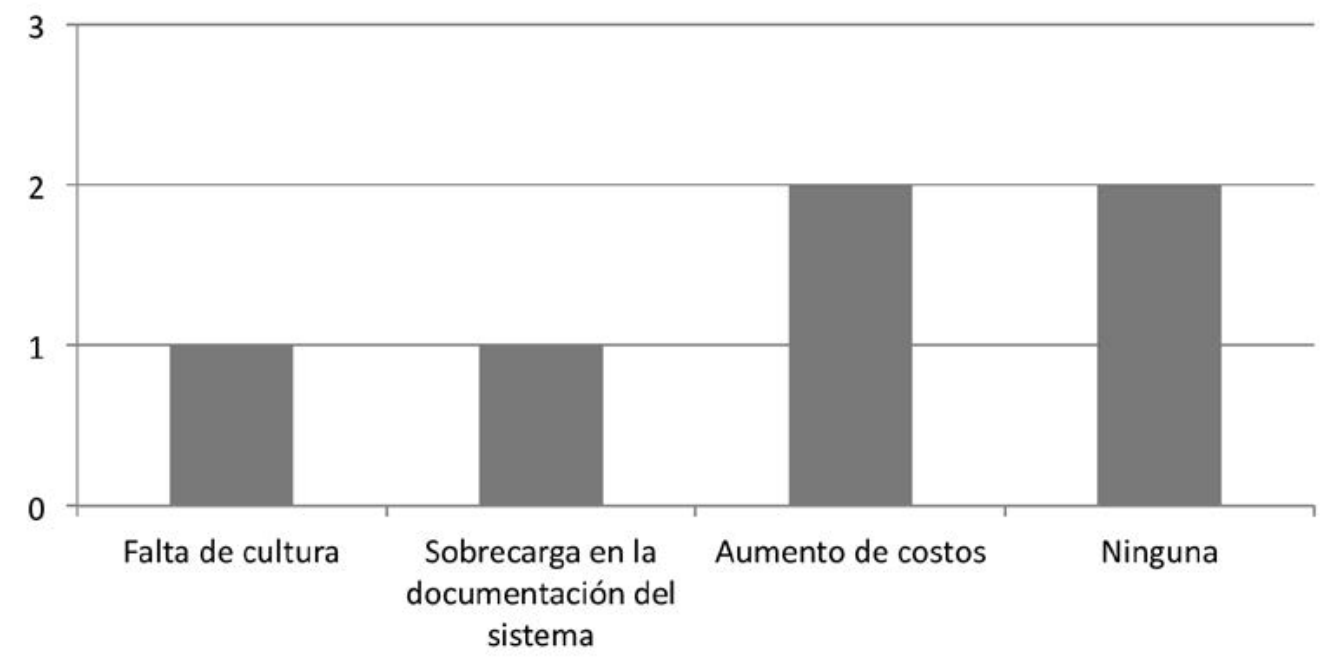

Fuente: autor.

Figura 7. ¿Se han llevado a cabo auditorías con enfoque integral?

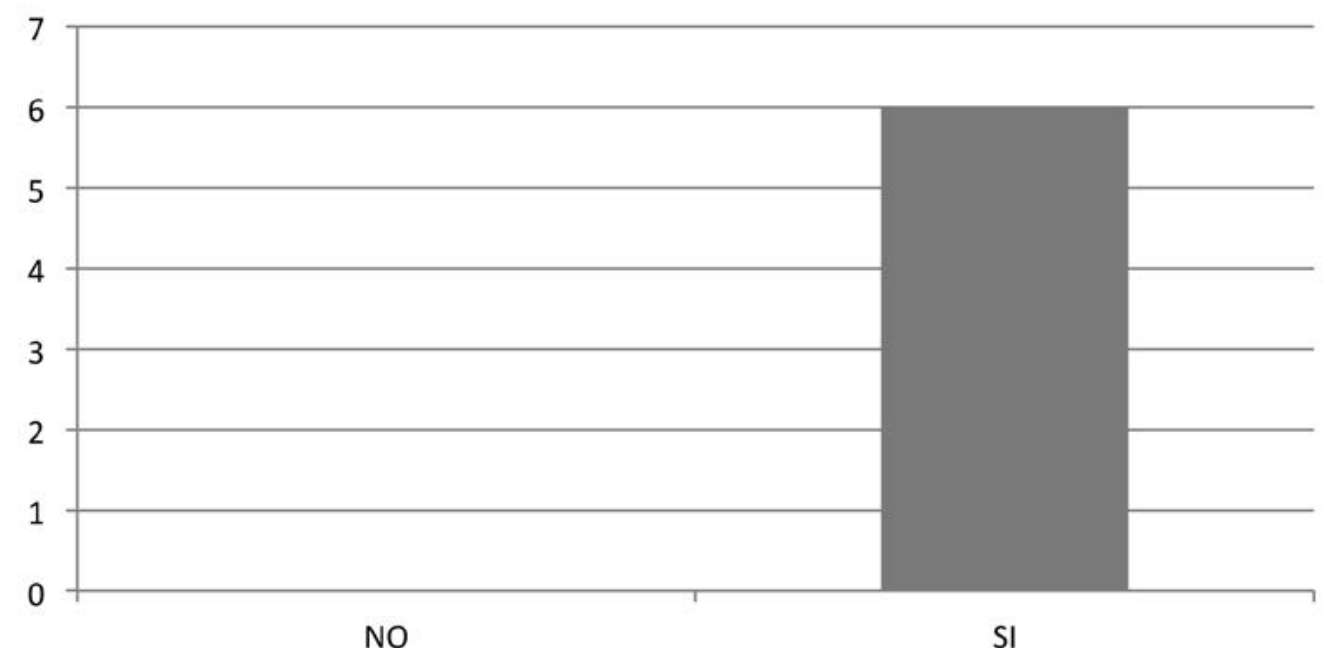

Fuente: autor. 
Según la figura 7, las seis empresas han realizado auditorías con enfoque integral.

Con respecto a las acciones de mejora que han encontrado en el sistema integrado de gestión, de acuerdo a la figura 8 , dos empresas refirieron que la acción de mejora ha sido la reducción de costos, una más mencionó una mayor participación gerencial, otra dijo que la revisión periódica del sistema de gestión, otra más mencionó la formalización de los canales de comunicación y la última nombró la generación de los programas de gestión.

Figura 8. Acciones de mejora en el sistema integrado de gestión

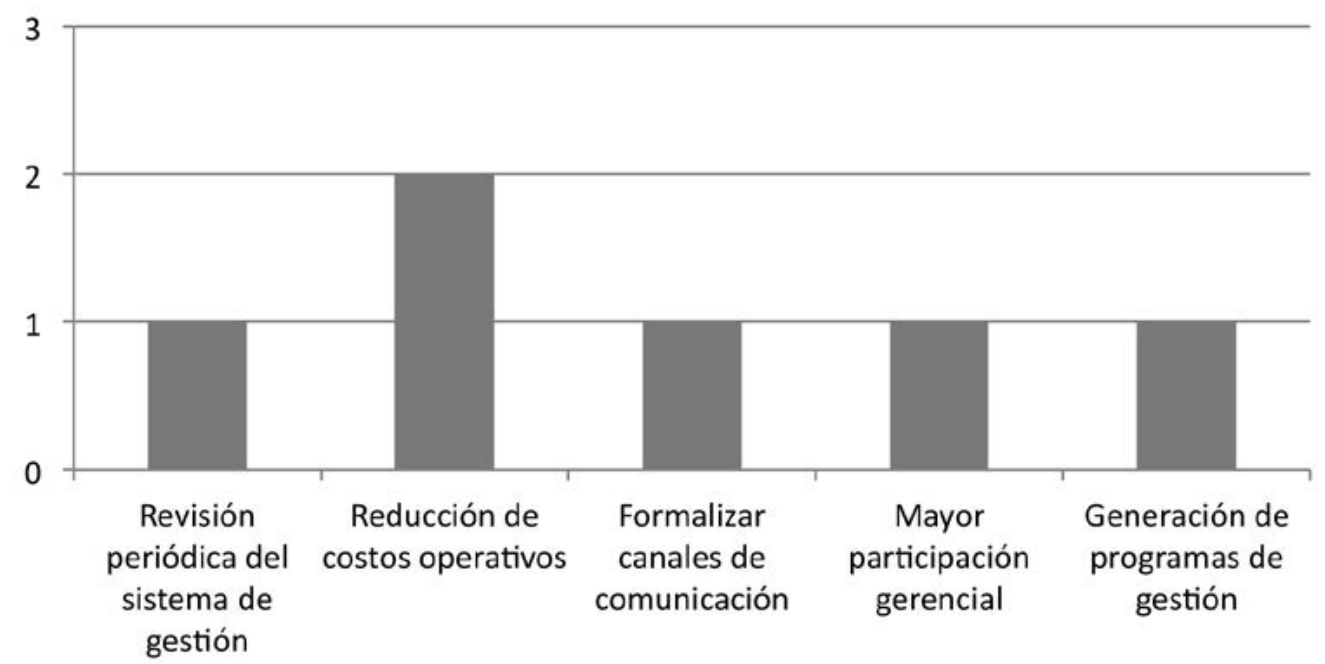

Fuente: autor.

Figura 9. ¿Qué percepción tiene la gerencia con respecto a los resultados del sistema integrado de gestión?

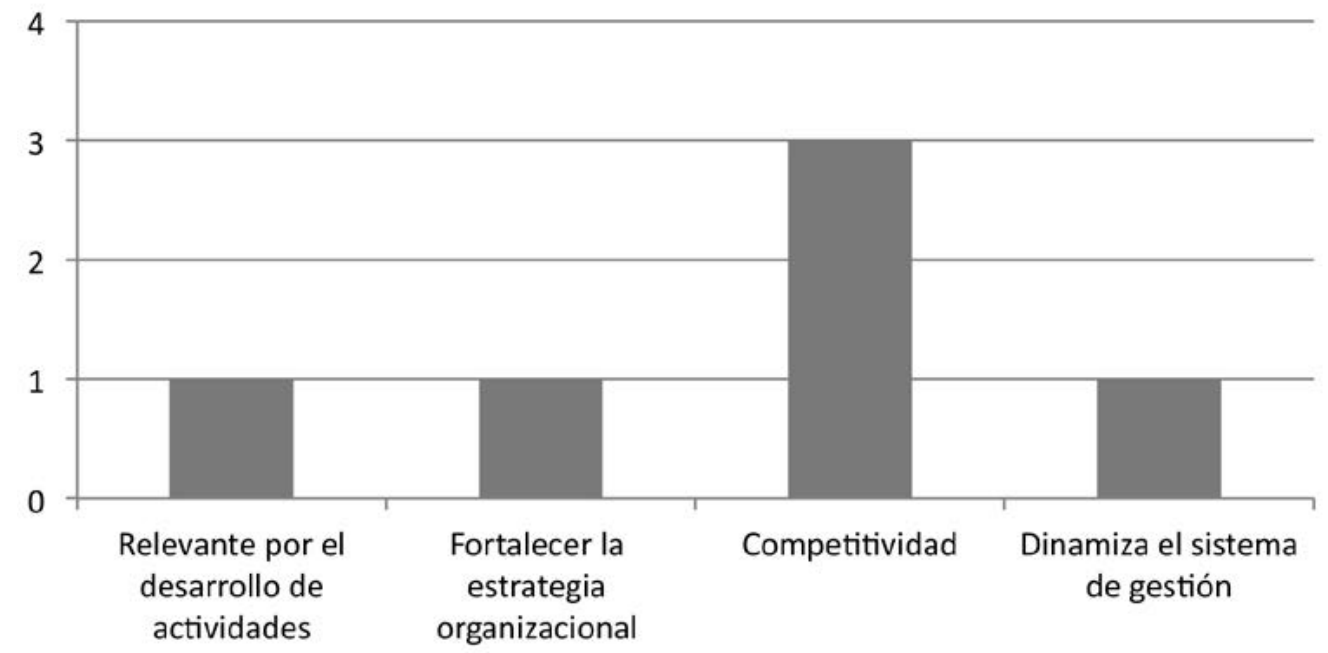

Fuente: autor. 
A la pregunta ¿qué percepción tiene la gerencia con respecto a los resultados del sistema integrado de gestión?, según la figura 9, la mayor percepción es la competitividad.

\section{Análisis de resultados del diaǵnóstico}

$\mathrm{Al}$ revisar los resultados del diagnóstico, se puede ver que las empresas tomadas en la muestra, las cuales tienen certificado sistemas de gestión, han decidido integrar sus sistemas (figura 1) sin realizar un análisis previo o de gestión de cambio. Se observa, según los resultados a la pregunta "¿qué motivo llevó a la organización a la integración de los sistemas de gestión?, que la mayor motivación se debió a requisitos de cliente (figura 2) y que el modelo más utilizado es el ciclo PHVA (figura 3),. Sin embargo, conforme a los resultados evidenciados en la figura 5, no hay una diferenciación importante en las ventajas de la integración, ya que se hizo más referencia en dos aspectos: el aprovechamiento de los recursos y la mejora en la organización de la empresa.

Pero esto no es coherente con los resultados obtenidos de la pregunta "¿qué desventajas se pueden generar de la implementación de la integración de los sistemas de gestión?" (figura 6) con respecto a las desventajas, pues el mayor aspecto referenciado fue el aumento de costos. Además, a la pregunta sobre las acciones de mejora que se han encontrado en el sistema integrado de gestión (figura 8), las empresas hicieron más énfasis en la reducción de costos, lo que permite ver que no son claros los beneficios de la integración de los sistemas de gestión, a pesar de que todas las empresas han realizado auditoría con enfoque integral (figura 7), la cual es un beneficio significativo.
Otro aspecto que se puede analizar de estos resultados es que no en todas las empresas el sistema de gestión está alineado con la planeación estratégica, razón por la cual no son claros los beneficios ni el aporte de los sistemas a los resultados de la organización.

Con relación a la percepción que tiene la gerencia respecto a los resultados del sistema integrado de gestión (figura 9), la mayoría se refirió a la competitividad, y esto se relaciona con la capacidad de generar mayor satisfacción al cliente. Y a la pregunta ¿qué motivo llevó a la organización a la integración de los sistemas de gestión?, la mayoría respondió que los requisitos exigidos por los clientes generan la motivación al momento de la integración (figura 2).

\section{Instrumento para la integración de los sistemas de gestión}

Teniendo en cuenta los resultados del diagnóstico anterior, en el que el liderazgo y el análisis de entorno fueron reconocidos como los factores claves de éxito para la integración de los sistemas de gestión en las empresas de servicios (aspectos que son valorados por los clientes (operadoras) del sector hidrocarburos), se plantea conceptualmente el modelo de integración denominado GOLD (gestión organizacional con liderazgo directivo), el cual se fundamenta en el ciclo de mejora y busca ser un modelo que aporte a fortalecer el análisis de contexto y la estructuración y planificación del sistema integrado de gestión.

El modelo GOLD (figura 10) puede amoldarse a las circunstancias que se desarrollan en cada organización que preste servicios y debe estar en función de sus políticas, objetivos, entorno y madurez. 
Fiǵura 10. Modelo GOLD

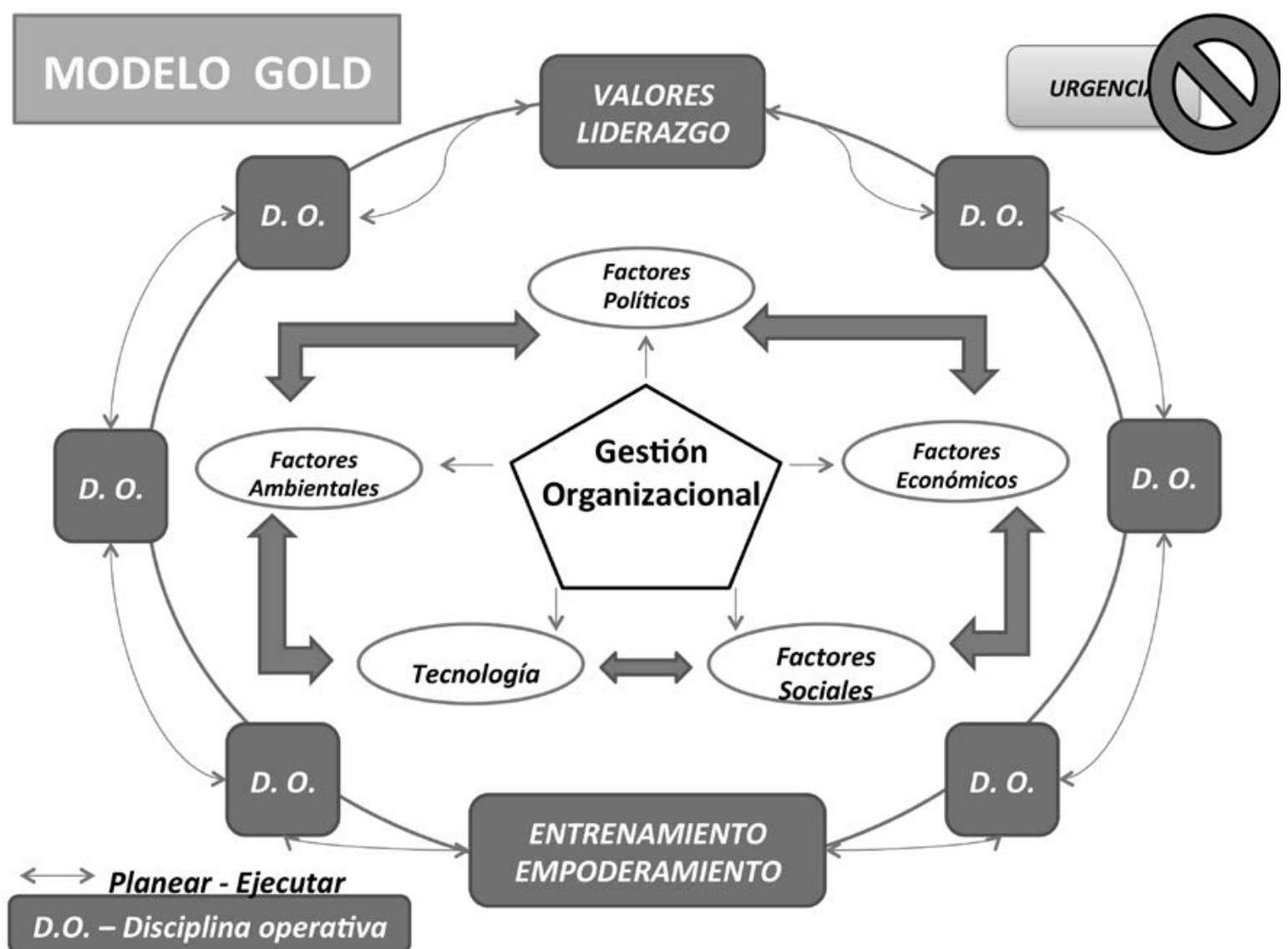

Fuente: autor.

Para implementar el modelo GOLD, se deben documentar los siguientes aspectos:

- Beneficios esperados: Analizar el(los) beneficio(s) de la integración, partiendo del hecho de que la integración de los sistemas alcanza a varias áreas de la organización, puede conllevar cambios funcionales, se requieren recursos y se debe definir el tiempo de esta actividad; por lo tanto, la organización debe identificar claramente los beneficios que espera alcanzar y los recursos que necesitará invertir.

- Análisis de entorno: Se requiere realizar una evaluación inicial o preliminar de la empresa y de los sistemas de gestión implementados para definir la estrategia organizacional. El conocimiento de la cultura organizativa, de su situación de partida en cuanto a la implementación de los sistemas de gestión y de las prioridades marcadas por la dirección son bases esenciales que hay que estudiar y valorar.

- Método de integración: Este aspecto se refiere a la documentación de las actividades del modelo de mejoramiento continuo seleccionado por ser resultado del diagnóstico.

- Compromiso alta dirección: El apoyo y el liderazgo de la alta dirección se plasman en acciones concretas, como la asignación de los recursos necesarios para llevar a cabo el plan de trabajo aprobado 
y su posterior seguimiento. A la alta dirección le compete nombrar al responsable o coordinador del modelo de integración.

\section{Implementación del modelo GOLD}

La implementación de este modelo consiste en responder a la pregunta ¿cómo se va a hacer 0 a ejecutar la implementación del modelo?, por lo cual se deben establecer los siguientes aspectos:

- Equipo encargado del modelo de integración de sistemas de gestión. Es necesario y conveniente conformar un equipo de integración del cual formen parte los responsables de los distintos departamentos o procesos que se van a integrar.

- Seguimiento del modelo de integración, con una frecuencia que permita controlar el cumplimiento de los objetivos fijados y, en el caso de desviaciones, introducir los oportunos cambios en el plan, actualizándolo. El seguimiento debe documentarse en informes de revisión que deben distribuirse a todas las partes implicadas en el proceso de integración y que han de tener presente los lineamientos especificados en el Anexo $\mathrm{H}$.

\section{Revisión y mejora del modelo GOLD}

El modelo adopta la filosofía de mejora continua siguiendo el ciclo PHVA. Esta última fase pretende responder a dos preguntas: ¿es eficaz y rentable la integración adoptada? y ¿necesita mejoras? Para responder a las anteriores preguntas, se recomienda realizar la revisión de manera conjunta y no una revisión individual, básicamente por los siguientes beneficios: se puede realizar una visión global de los resultados, la mejora se enfoca en las decisiones gerenciales y debe ser coherente con estas, de ahí la importancia de su liderazgo y de la determinación de las prioridades de acuerdo con los resultados en los distintos elementos del sistema integrado de gestión.

\section{CONCLUSIONES}

Este proyecto permitió revisar las características del sector de los hidrocarburos desde las empresas que le prestan servicios. Se concluye que es una industria pionera en la introducción de métodos de control, que se caracteriza por requerir una fuerza de trabajo especializada que cuente con equipos de avanzada tecnología y cuyas empresas incorporen valor agrado a sus servicios, ya que en sus procesos de selección exigen una planeación seria que permita hacer un buen análisis de los riesgos a los cuales pueden estar expuestos y que buscan las mejores garantías.

Con respecto al sector de los hidrocarburos, la necesidad de servicios creció debido al aumento de la producción de petróleo y del gas, la cual se incrementó un $49 \%$ y un $77 \%$ respectivamente en Colombia en los últimos seis años, esto, en parte, gracias a la gestión de la Agencia Nacional de Hidrocarburos (ANH). Adicionalmente, la industria pasó de representar el $11,3 \%$ del PIB del sector de hidrocarburos en 2004 a tener una participación del 20,9\% en 200, siendo la inversión extranjera directa (IED) uno de los factores que más ha incidido en el crecimiento del sector debido al aumento de la participación privada en las actividades petroleras y al fomento de la IED. La participación del sector petrolero en la IED total aumentó $14 \%$ de 2003 a 2013, pasando del 16 al $30 \%$.

El sector de servicios petroleros en el país está concentrado principalmente en servicios relacionados con la perforación, el suministro de maquinaria y equipo, servicios de consultoría e ingeniería y servicios de geología, entre otros. El $23 \%$ de los ingresos operacionales que genera el sector de servicios petroleros 
corresponde a empresas que prestan servicios de perforación, completamiento y workover, mientras que 22 $\%$ de los ingresos corresponde a empresas proveedoras de maquinaria, equipos y herramientas.

Como conclusión del primer objetivo específico y de la fase 1, al aplicar el análisis PESTA a la empresa MARENFOX S. A., se observa que hay una débil integración de los sistemas de gestión; que es un servicio con permanente regulación por parte de la Agencia Nacional de Hidrocarburos (ANH) y del Ministerio de Ambiente y Desarrollo Sostenible; que en el desarrollo de cada uno de los proyectos deben dar apoyo (trabajo) a las comunidades cercanas; y que a nivel de tecnología, requieren equipos con altos estándares de seguridad. Estos resultados pueden extrapolarse a las empresas de la muestra al realizar el análisis de entorno en cada una.

Con respecto al segundo objetivo específico, se analizaron los requerimientos de los dos más grandes contratantes del país, es decir, Ecopetrol y Pacific Rubiales Energy, y se concluye que Ecopetrol, en su Manual de contratación, define los requisitos de calidad, seguridad, salud y ambiente; además, presenta las normas y los procedimientos bajo los cuales se celebran los procesos de selección de contratistas. Con respecto a Pacific Rubiales Energy, la empresa define los requisitos en el Anexo HSEQ, el cual deben cumplir los contratistas y sus subcontratistas, requisitos que forman parte integral del contrato u orden de servicios y que se deben ejecutar manteniendo las directrices de las normas ISO 9001, ISO 14001 y OHSAS 18001.

Este proyecto constituye un aporte para el diagnóstico de la integración en empresas certificadas, ya que, como conclusión del análisis realizado, se pudo evidenciar que las organizaciones analizadas no han realizado un análisis de entorno que permita fortalecer la definición de la misión, visión y la política del sistema de gestión y, por ende, el establecimiento de objetivos y metas.

Según los resultados del diagnóstico, se puede concluir que la mayor motivación para la integración de los sistemas de gestión se debió a requisitos de cliente (figura 6) y que no hay una diferenciación importante en las ventajas de esta integración (figura 9), por lo que el modelo GOLD de integración plantea iniciar con el análisis de los beneficios de la integración, partiendo del hecho de que la integración de los sistemas alcanza a varias áreas de la organización.

Se concluye, además, que es necesaria una actividad permanente de sensibilización a trabajadores de las empresas para mejorar la comunicación y la divulgación, dado que hay un riesgo probable de perder las certificaciones, lo que afectará la prestación del servicio, y esto conlleva una pérdida de imagen y de clientes.

Como conclusión del cuarto objetivo específico, y tomando en cuenta los resultados del diagnóstico —que finaliza indagando sobre la percepción que tiene la gerencia con respecto a los resultados del sistema integrado de gestión-, de acuerdo a la figura 13, el $50 \%$ de las empresas objeto de estudio se refirió a la competitividad, entonces, se plantea el modelo GOLD (gestión organizacional con liderazgo directivo), el cual se fundamenta en el liderazgo y análisis de entorno como factores claves de éxito para la integración, modelo en el que se explica que la evolución del liderazgo en el sector servicios (la pirámide de liderazgo) funciona de manera invertida buscando brindar herramientas para cada uno de los funcionarios operativos, de tal manera que se empoderen y se pueda ofrecer un buen servicio al cliente y a las partes interesadas.

Con respecto a la hipótesis planteada en la definición del problema, y teniendo en cuenta los resultados del diagnóstico realizado a las empresas de servicios, se 
puede concluir que la integración de los sistemas de gestión en las empresas de servicios del sector hidrocarburos puede generar una mayor optimización de los recursos al gestionar los diferentes sistemas implementados y hacerlas más competitivas.

\section{RECOMENDACIONES}

Se sugiere fortalecer el análisis y la divulgación de los beneficios de la integración de los sistemas de gestión para que cada área de las organizaciones pueda manejar los cambios funcionales que se requieren y se definan los tiempos y recursos que se necesitarán para gestionar la integración de los sistemas de gestión.

Se recomienda la definición de unas buenas competencias para el equipo encargado de la integración de sistemas de gestión, de tal manera que se puedan ir cumpliendo las actividades con disciplina operativa y fundamentándose en los valores que plantea el modelo GOLD.

Es importante conocer los requisitos de las normas ISO 9001 e ISO 14001, versiones 2015, para hacer el análisis de gestión organizacional y su correspondiente implementación.

Se sugiere analizar otros instrumentos para gestionar la integración y poder tener más elementos de análisis al momento de plantear sus beneficios.

Este proyecto hizo el análisis para empresas de servicios; se recomienda realizar análisis para empresas de bienes o productos y analizar aspectos tales como la sinergia entre los sistemas, la gestión documental, los costos operativos al compartirse gastos, la formación del personal debida a la integración de procesos, la percepción e implicación del personal en los sistemas de gestión y la capacidad de reacción de la organización frente a las nuevas necesidades o expectativas de las partes interesadas.

\section{REFERENCIAS}

Bernal, C. A. (2006). Metodología de la investigación (2.a ed.). México: Pearson Educación.

Ecopetrol. (2012). Manual de contratación de Ecopetrol S. A. Versión 1. Recuperado de http://www.ecopetrol. com.co/documentos/38390_Manual_de_Contratacion-15-10-12.pdf

Ecopetrol. (2013). Gestión de contratistas - Foco HSE. Versión 2. Recuperado de http://www.ecopetrol. com.co/documentos/80118_FOCO_19-04-2013. pdf

Fedesarrollo. (julio de 2015). Informe de Coyuntura Petrolera. Bogotá: Autor. Recuperado de goo.gl/ by $5 \mathrm{DpN}$

Friedman, M. (2009). Integrated Management System. Proceedings of World Academy of Science. Engineering \&̊ Technology, 51, 734-739.

Hulley, S. y Cummings, S. (1993). Diseño de la investigación clínica. Barcelona: Ed. Doyma.

Instituto Colombiano de Normas Técnicas y Certificación - ICONTEC. (2009). Sistemas de gestión de la calidad: Fundamentos y vocabulario. NTC - ISO 9000. Bogotá: ICONTEC.

(2008). Sistemas de gestión de la calidad. Requisitos. NTC - ISO 9001. Bogotá: ICONTEC.

(2007). Sistemas de gestión en seguridad y salud 
ocupacional. Requisitos. NTC-OHSAS 18001. Bogotá: ICONTEC.

(2004). Sistemas de gestión ambiental. Requisitos con orientación para su uso. NTC-ISO 14001. Bogotá: ICONTEC.

Pacific Rubiales Energy. Anexo HSEQ para contratistas (en línea). Recuperado de https://goo.g//AdNZIH
Peña, G. y Tejada, F. (2009). Reflexiones sobre las características constitutivas de la gestión integral. Revista Signos,1(2), 79-93.

Unidad de Planeación Minero Energética. (2015). Evaluación de la contribución económica del sector de hidrocarburos colombiano. Bogotá: Subdirección de Hidrocarburos UPME. Recuperado de goo.gl/ yQxXtS 